\title{
Publisher's Note: Magnetic Interaction between Surface-Engineered Rare-Earth Atomic Spins [Phys. Rev. X 2, 021012 (2012)]
}

\author{
Chiung-Yuan Lin, Jheng-Lian Li, Yao-Hsien Hsieh, Keng-Liang Ou, and B. A. Jones \\ (Received 18 July 2012; published 26 July 2012)
}

DOI: 10.1103/PhysRevX.2.039902

Subject Areas: Magnetism, Nanophysics

This paper was published online on 22 June 2012 with an incorrect ordering of author names in the author and affiliation lists, as well as an error in the Acknowledgments. The correct ordering of author names is Chiung-Yuan Lin, Jheng-Lian Li, Yao-Hsien Hsieh, Keng-Liang Ou, and B. A. Jones. The correct affiliations for the fourth and fifth authors are KengLiang Ou: Research Center for Biomedical Devices, Taipei Medical University, Taipei, Taiwan, and College of Oral Medicine, Taipei Medical University, Taipei, Taiwan; B. A. Jones: IBM Almaden Research Center, San Jose, California 95120-6099, USA. The second sentence in the Acknowledgments on page 8 should read as "C.-Y.L. acknowledges financial support from the Taiwan National Science Council (under Grant No. NSC 97-2112-M-009-007-MY3), National Chiao Tung University Ministry-of-Education Aiming-for-Top-University Program, and Taiwan National Center for Theoretical Sciences, and facility support from the Taiwan National Center for High-performance Computing." The paper has been corrected online as of 20 July 2012.

Published by the American Physical Society under the terms of the Creative Commons Attribution 3.0 License. Further distribution of this work must maintain attribution to the author(s) and the published article's title, journal citation, and DOI. 